\title{
Development of high-throughput, polarization-maintaining, near-field probes
}

\author{
Vivekananda P. Adiga, Paul W. Kolb, Geoffrey T. Evans, Max A. Cubillos-Moraga, \\ Don C. Schmadel, Richard Dyott, and H. Dennis Drew
}

\begin{abstract}
A novel, chemical-etching technique produces very high throughput, polarization-maintaining probes for near-field, scanning, optical microscopy (NSOM). The process includes coating the tips with aluminum and forming the apertures with a focused ion beam (FIB). The elliptical core fibers used resulted in elliptical apertures for the probes. The throughput of the probes depends on the incident polarization. For polarization parallel to the minor axis, the tip presents an insertion loss of only $20 \mathrm{~dB}$ for aperture widths of $55 \mathrm{~nm}$. Probes have a typical polarization extinction of 100 to 1 in the far field. These tips produced NSOM images of gold dots on a GaAs substrate in reflection mode. (C) 2006 Optical Society of America OCIS codes: $180.5810,230.4000,110.1220$.
\end{abstract}

\section{Introduction}

NSOM utilizes a subwavelength aperture, which allows spatial resolution surpassing the diffraction limit. ${ }^{1-3}$ The aperture, an important determinative component of NSOM, has usually been formed on the end of an optical fiber, which has been tapered by mechanical pulling 4 or chemical etching and then coated with aluminum. ${ }^{5}$ In the current work, we adapted chemical etching to our needs, because it better preserves the propagation of the core and therefore yields a higher throughput for a given aperture size. This enhances the utility of NSOM in experiments such as near-field Raman spectroscopy ${ }^{6}$ and single molecule fluorescence. ${ }^{7}$

A critical consideration in interpreting NSOM images is the polarization of the illumination, which, among its other effects, is one of the factors influencing the optical contrast. ${ }^{8-10}$ In a conventional circular core optical fiber, it is very difficult to control the

V. P. Adiga is with the Department of Materials Science and Engineering, University of Maryland, College Park, Maryland 20742. P. W. Kolb is with the Laboratory for Physical Sciences, 8050 Greenmead Drive, College Park, Maryland 20740. G. T. Evans, M. A. Cubillos-Moraga, D. C. Schmadel (schmadel@physics. umd.edu), and H. D. Drew are with the Department of Physics, University of Maryland, College Park, MD 20742. R. Dyott (deceased) was with KVH Industries, Tinley Park, Illinois 60477 when this research was performed.

Received 18 July 2005; revised 11 November 2005; accepted 16 November 2005; posted 17 November 2005 (Doc. ID 63480).

$0003-6935 / 06 / 122597-04 \$ 15.00 / 0$

(C) 2006 Optical Society of America polarization, which changes in response to stress and temperature-induced geometrical nonuniformities in the core-clad geometry. Therefore, we developed probes using optical fiber from KVH, Inc. which has an elliptical core, which splits the degeneracy of the two polarization modes and, hence, prevents energy exchange. Measurements of the probes developed from this fiber suggest unique optical transmission properties such as polarization-dependent throughput, good polarization maintenance, and very high throughput. The results compare quite favorably with tips produced from stress-induced birefringent fibers using heating and pulling. ${ }^{11,12}$

\section{Chemical Etching}

The particular optical fiber we used has three different regions, a Ge-doped core with major and minor axes of 1 and 2 microns, respectively, a surrounding fluorine-doped cladding with an outer circular diameter of 10 microns, and a pure silica outer cladding. Additionally, the fiber has a polymer jacket that attenuates the cladding modes to below the detector noise level within a few inches of travel. The fiber also possesses a manufacturing artifact: an underdoped region of about $50 \mathrm{~nm}$ diameter in the center of the core. ${ }^{13}$ Even though this artifact does not significantly affect the optical transmission properties of the fiber, it does cause a deep and narrow crater to form in the center of the tip during the chemicaletching process. To eliminate this crater formation, we homogenize the end of the fiber by heating it to approximately $1650{ }^{\circ} \mathrm{C}$ for two minutes. Fibers are 


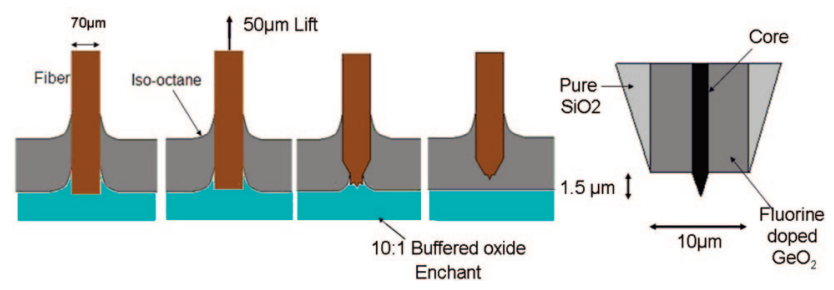

Fig. 1. (Color online) Schematic diagram of the etching process. The core of the fiber is tapered down by selective-chemical etching. After 10 hours, the meniscus will drop down when it starts etching the fluorine-doped cladding. The drawing on the right illustrates the end result.

suspended vertically during the heating to avoid bending at the high temperature.

Our chemical-etching process uses a combination of selective-etching ${ }^{14}$ and meniscus-etching ${ }^{15}$ techniques. Selective chemical etching creates smooth sharp tips with elliptical apices, while meniscus etching tapers the surrounding cladding. Figure 1 illustrates the process, which involves 10:1 buffered oxide etchant (BOE) covered with a $1 \mathrm{~cm}$ layer of isooctane to prevent evaporation of the $\mathrm{BOE}$ and to protect the exposed fiber cladding above the etch. The very end of the cleaved fiber is lowered until it just contacts the isooctane-BOE interface and forms a meniscus. It is then vertically raised and held 25 microns (larger diameter fibers will generally require greater distances) from the position of initial contact. As the etching proceeds, the core begins to protrude beyond the claddings because the core of the fiber etches at a slower rate. Ultimately, an elliptical cone develops having an apex with a radius of curvature of $100 \mathrm{~nm}$ about the major axis and a radius of curvature of $20 \mathrm{~nm}$ about the minor axis. Also, as the etching continues, the height of the meniscus slowly drops as the radius of the fiber decreases. When it reaches the end of the fiber, the meniscus breaks away from the fiber and the etching ceases. The overall profile of the probe is shown at the right of Fig. 1. Because the fiber had been raised 25 microns from the initial point of contact with the BOE, as described above, the meniscus drops before the meniscus interface reaches the core. Thus, the geometry of the tapered-core tip is entirely determined by the dynamics of the selective etching rather than the less predictable dynamics of the meniscus interface. As shown in the SEM image at the top of Fig. 2, the plateau surrounding the tip is not circular, which might result from the intrinsic stresses in the fiber induced during the manufacturing process. Also apparent in the figure, a somewhat undesirable, broad and shallow crater surrounding the protruding core results from the slightly faster etching rate of the fluorine-doped cladding as compared to the pure Si outer cladding.

The etched fibers are next coated with aluminum by thermal evaporation. An aperture is formed by slicing off the very end of the coated probe using focused ion beam (FIB) ${ }^{16,17}$ milling with a typical beam intensity of $30 \mathrm{kV}$ and $11 \mathrm{pA}$. The lower picture in Fig. 2 shows the resulting $55 \mathrm{~nm} \times 225 \mathrm{~nm}$ aper-

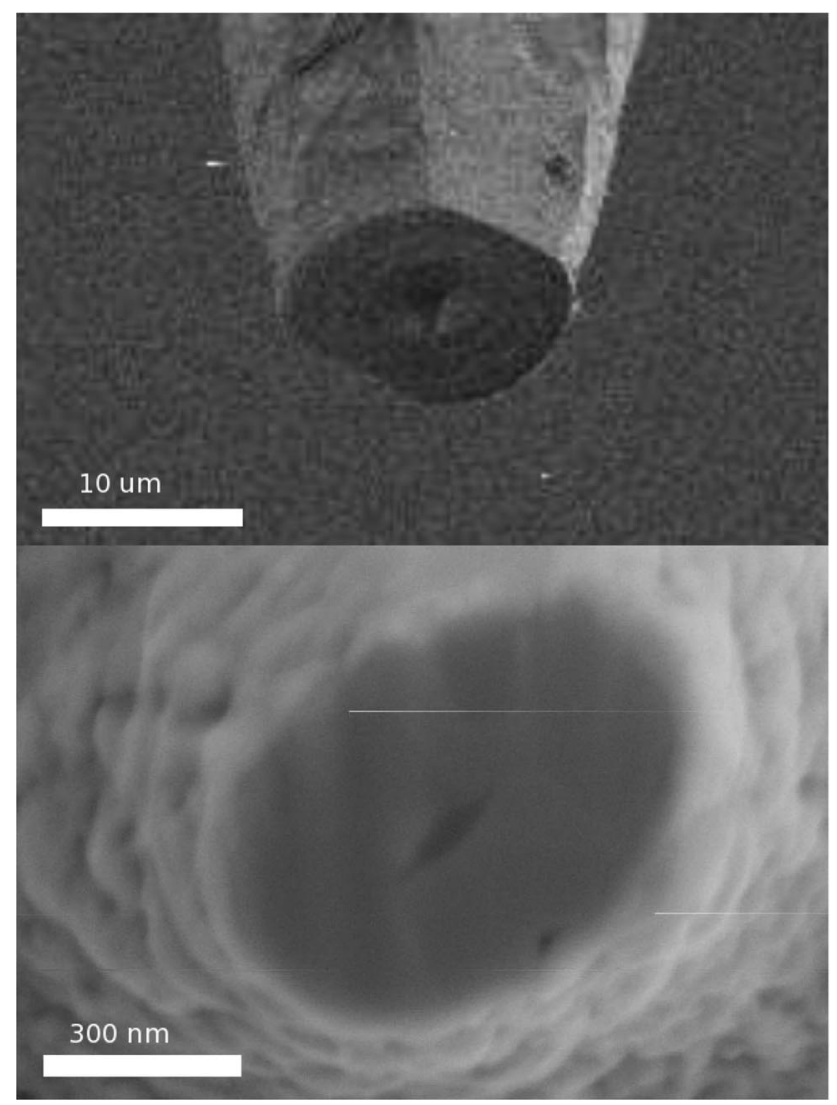

Fig. 2. (top) SEM image of the uncoated probe. Preferential etching leads to a noncircular plateau. (bottom) An SEM image of the tip after FIB milling. The aperture has dimensions of about $55 \pm$ $5 \mathrm{~nm}$ by $225 \pm 5 \mathrm{~nm}$.

ture of a fiber tip that has been coated with $300 \mathrm{~nm}$ of aluminum to ensure that the measured throughput is in fact from the probe end and not leakage through pinholes. For normal use the probes would be coated with less than 100 of aluminum.

\section{Characterization of the Probes}

\section{A. Far-Field Throughput of the Probes}

Our first characterization arrangement directly launches $635 \mathrm{~nm}$ radiation from an Hitachi HL6320G laser diode into the input end of a fiber without any intervening optics. The arrangement permits rotation of the input polarization. A detector at a distance of $1 \mathrm{~mm}$ from the output end collects the transmitted, far-field light. Using this arrangement, the throughput of a length of elliptical core fiber cleaved at both ends proves to be independent of polarization and typically $20 \mu \mathrm{W}$ with a margin of error of $15 \%$ when the distance between laser and fiber is approximately $150 \mu \mathrm{m}$. For each measurement, the output is always maximized by adjusting the lateral position of the fiber while maintaining the distance between the fiber and the laser constant. Referenced to a cleaved, polarization-maintaining fiber, Fig. 3 (top) shows the output of a fiber probe with an aluminum-coated tip at the output end and with the input radiation ini- 

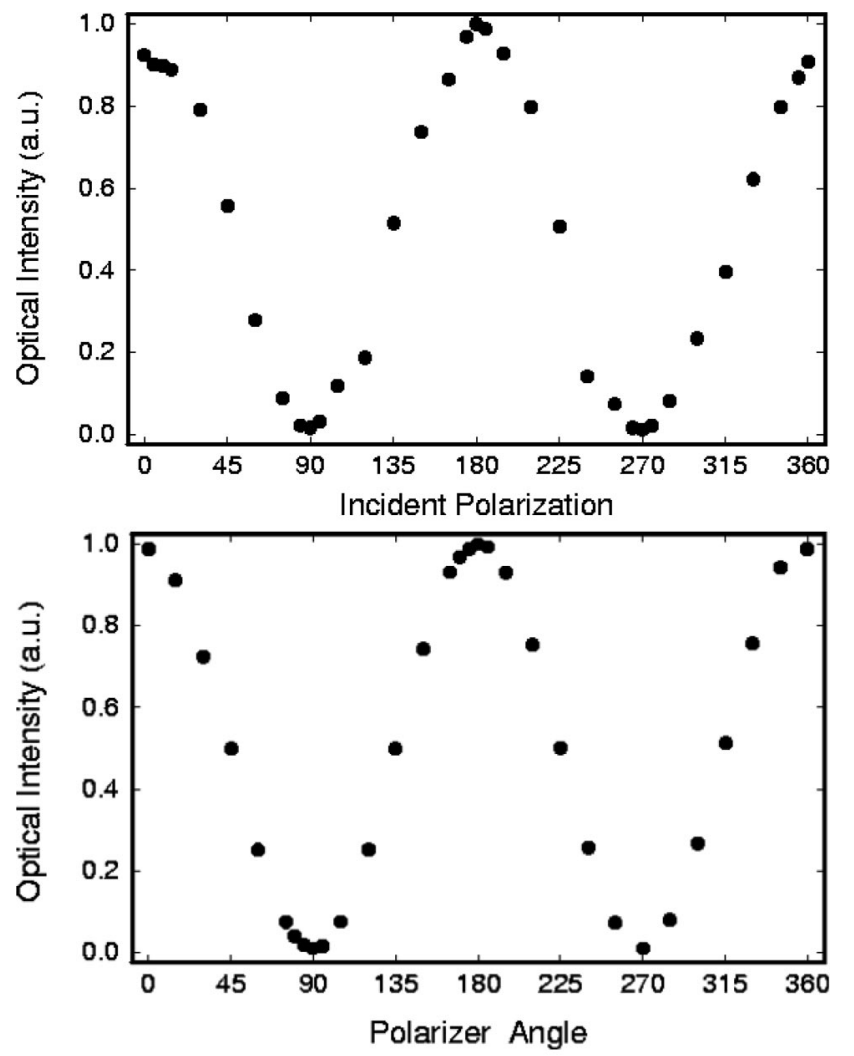

Fig. 3. (top) Output intensity of the probe shown in Fig. 2 as a function of input polarization. (bottom) The polarization extinction of the same probe for a fixed input polarization.

tially along the minor axis. Remarkably, the results also show the throughput loss for a $55 \mathrm{~nm}$ probe to be only $20 \mathrm{~dB}$ as compared to $32 \mathrm{~dB}$ for tips made by heating and pulling stress-induced birefringent fiber. ${ }^{11,12}$

The measured behavior or the probe fiber tip is also similar to that of a polarizer with an extinction ratio of 100 . In the simplest case, one may understand this by first recalling that the input light splits into two components: one along major axis of the elliptical core and one along the minor axis. These components travel at different velocities, so the output of a fiber with a cleaved end is generally elliptically polarized. However, the aluminum-coated tapered ends of our fiber probes, having subwavelength elliptical apertures, attenuate the component with polarization parallel to the major axis much more than the component parallel to the minor axis because of the boundary conditions imposed by the metal cladding. ${ }^{18}$ Therefore,

$$
T=T_{\|} \cos ^{2} \theta+T_{\perp} \sin ^{2} \theta,
$$

with $T_{\perp} \ll T_{\|}$, where $T_{\perp}$ and $T_{\|}$are the power transmissions for polarizations perpendicular and parallel to the minor axis, respectively. In essence, the probe acts like a polarizer with the polarization axis along the minor axis for very small apertures. Maxima and minima from these measurements are used for throughput calculations summarized in Fig. 4, which

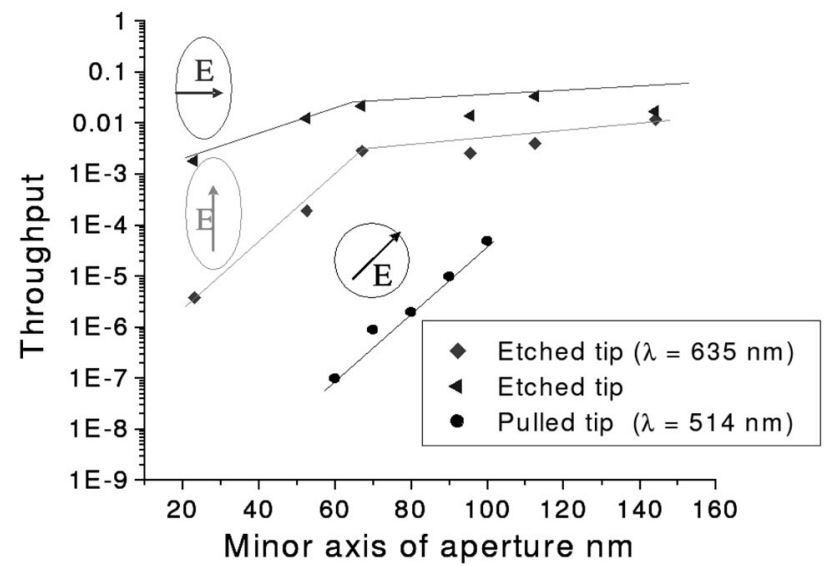

Fig. 4. Throughput as a function of the minor-axis dimension. The aspect ratios of the tips were around 4.5 except for the probe with minor axis dimension of $144 \mathrm{~nm}$, which had an aspect ratio of about 3.25.

shows results for fiber tips with minor-axis dimensions from $23 \mathrm{~nm}$ to $150 \mathrm{~nm}$. The throughputs reported here are much higher than the reported throughputs of pulled and etched tips for the comparable aperture dimensions. This is due to the elliptical shape of our apertures and the higher cone angle of the tapered core, which reduces the transmission loss through the metallic portion of the waveguide.

\section{B. Polarization Maintenance}

Our second characterization arrangement examines the polarization of the output of the probe ends by placing a polarizer between the aluminum-coated tip and the detector. Figure 3 (bottom) shows the result for the case when the input polarization was along the minor axis of the fiber. Again this follows a cosinesquared behavior, indicating the output to be polarized along the minor axis of the aluminum-coated tip. Next the input polarization was rotated to be along the major axis of the fiber. This also followed a cosinesquared behavior but with much less output power and with the maxima along the major axis of the aluminum-coated tip indicating the polarization had rotated $90^{\circ}$. These results suggest that the fiber probes will maintain polarization so as to permit their use in obtaining scanning data for both polarizations without rotating the tip with respect to the sample.

\section{Near-Field Scanning Optical Microscopy Imaging}

We tested one of these probes in a NSOM microscope in reflection mode in which we imaged a sample of gold dots on a GaAs substrate. The NSOM tip used had a minor and major axis aperture of dimensions of $23 \pm 5 \mathrm{~nm}$ by $100 \pm 5 \mathrm{~nm}$, respectively. A laser diode supplied the illumination, which was coupled to the cleaved end of the fiber with incident polarization along the minor axis so as to get higher throughput from the tip aperture. A microscope objective $(40 \times$, NA 0.4$)$ placed at about $30^{\circ}$ to the sample plane collected the light reflected from the sample. 


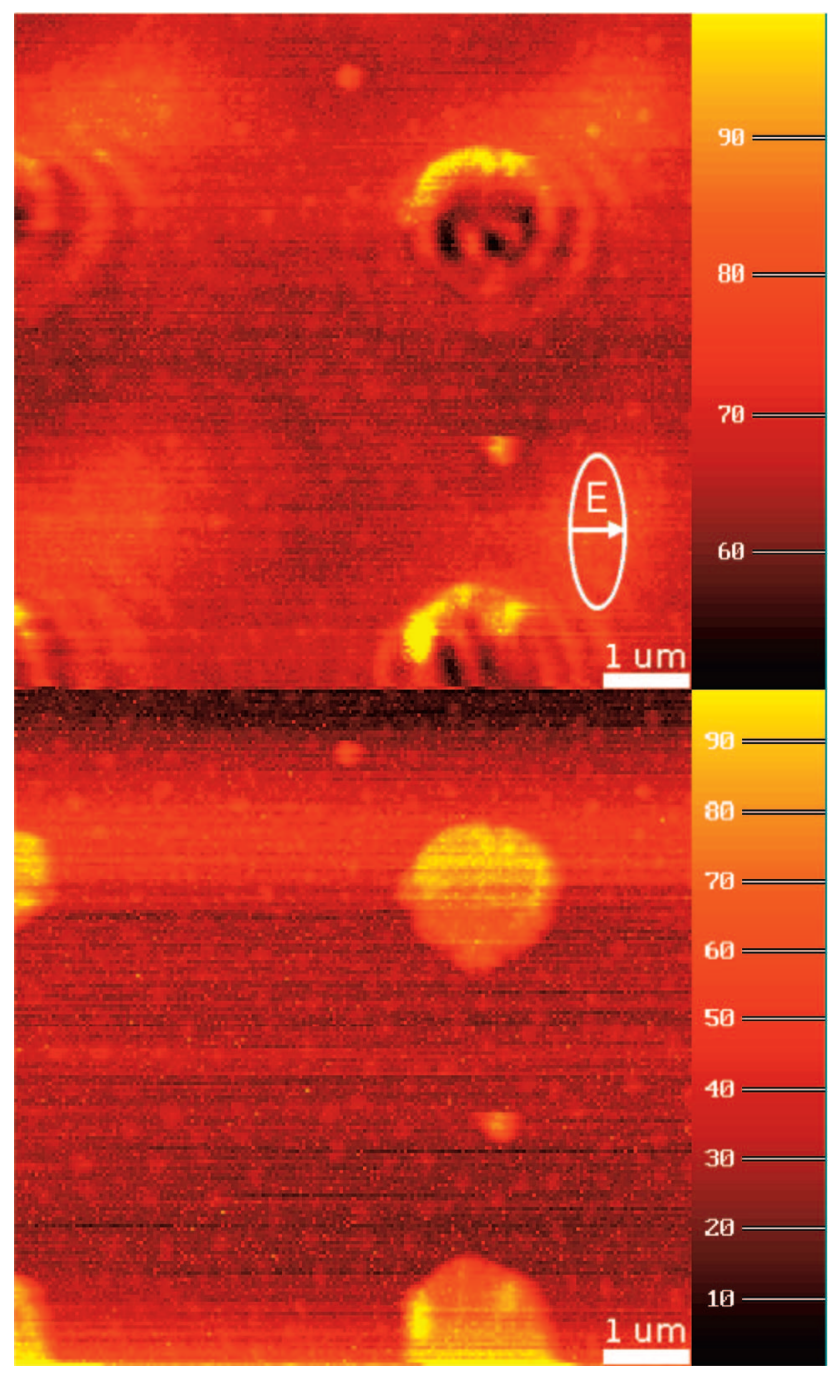

Fig. 5. (Color online) NSOM images of gold dots on a GaAs substrate. The top is an optical intensity image. The bottom is a topographic image.

The fiber probes are oriented with the minor axis of the probe perpendicular to the plane-containing probe and the detector so as to maximize the signal. ${ }^{10}$ This will induce dipoles in the sample that will radiate mostly in the plane-containing detector and the probe. Figures 5 (top) and 5 (bottom) show the resulting intensity and topographic scans of the gold dots of size $1.4 \mu \mathrm{m}$ and height $30 \mathrm{~nm}$. The topographydominated effects such as bright edges of the dots and the shadowing effects are readily apparent in the image. The contrast inversion of the gold dots could be due to the thick aluminum coating surrounding the tips. For instance, the tangential component of the electric field should be reduced by the boundary conditions imposed when the aluminum-coated tip is over a gold dot. But when the tip is over the GaAs substrate, this component is not so much restricted and so the reflected signal increases. Interference also appears in the scan of Fig. 5 (top) as rings sur- rounding the dots. They result from light from the tip interfering with light reflected from the dot. The preferential direction of the rings is due to the light being blocked either by the tip or the dot in the upper and lower side of the dot. Since the topographic effects dominate the optical response, the NSOM image does not demonstrate the high spatial resolution expected of the probes.

\section{References}

1. A. Lewis, M. Isaacson, A. Harootunian, and A. Murray, "Development of a 500-Å spatial-resolution light-microscope," Ultramicroscopy 13, 227-231 (1984).

2. D. Pohl, W. Denk, and M. Lanz, "Optical stethoscopy: image recording with resolution,” Appl. Phys. Lett. 44, 651-653 (1984).

3. E. Betzig, J. Trautman, T. Harris, J. Weiner, and R. L. Kostelak, "Breaking the diffraction barrier: optical microscopy on a nanometric scale," Science 251, 1468-1470 (1991).

4. G. A. Valaskovic, "Parameter control, characterization, and optimization in the fabrication of optical fiber near-field probes," Appl. Opt. 34, 1215-1228 (1995).

5. M. Ohtsu, Near-Field Nano/Atom Optics and Technology (Springer-Verlag, 1998).

6. V. Deckert, D. Ziesel, R. Zenobi, and T. Vo-Dinh, "Near-field surface-enhanced Raman of dye-labeled DNA with 100-nm resolution," Anal. Chem. 70, 2646-2650 (1998).

7. N. Hosaka and T. Saiki, "Near-field fluorescence imaging of single molecules with a resolution in the range of $10 \mathrm{~nm}$," J. Microsc. 202, 362-364 (2001).

8. E. B. McDaniel and J. W. P. Hsu, "Nanometer scale optical studies of twin domains and defects in lanthanum aluminate crystals," J. Appl. Phys. 80, 1085-1093 (1996).

9. E. Betzig, J. K. Trautman, J. S. Weiner, T. D. Harris, and R. Wolfe, "Polarization contrast in near-field scanning optical microscopy," Appl. Opt. 31, 4563-4568 (1992).

10. J. A. Cline and M. Isaacson, "Probe-sample interactions in reflection near-field scanning optical microscopy," Appl. Opt. 3, 4869-4876 (1995).

11. T. Mitsui and T. Sekiguchi, "Observation of polarization property in near-field optical imaging by a polarization-maintaining fiber probe," J. Electron Microsc. 53(2), 209-215 (2004).

12. T. Mitsui, "Development of a polarization-preserving opticalfiber probe for near-field scanning optical microscopy and the influences of bending and squeezing on the polarization properties," Rev. Sci. Instrum. 76, 043,703 (2005).

13. R. Dyott, Elliptical Fiber Waveguides (Artech House, 1995).

14. S. Monomode and M. Ohtsu, "Fabrication of pencil-shaped fiber probe for near-field optics by selective chemical etching," J. Lightwave Technol. 14, 2231-2235 (1996).

15. P. Hoffmann, B. Dutoit, and R. P. Salath, "Comparison of mechanically drawn and protection layer chemically etched optical fiber tips," Ultramicroscopy 61, 165-170 (1995).

16. S. Pilevar, K. Edinger, W. Atia, I. Smolyaninov, and C. Davis, "Focused ion-beam fabrication of fiber probes with well-defined apertures for use in near-field scanning optical microscopy," Appl. Phys. Lett. 72, 3133-3135 (1998).

17. J. A. Veerman, A. M. Otter, L. Kuipers, and N. F. van Hulst, "High definition aperture probes for near-field optical microscopy fabricated by focused ion beam milling," Appl. Phys. Lett. 72, 3115-3117 (1998).

18. A. Zakharian, M. Mansuripur, and J. Moloney, "Transmission of light through small elliptical apertures," Opt. Express 12, 2631-2648 (2004). 